\title{
Diversité floristique des communautés végétales dans l'espace de la Forêt des Marais Tanoé-Ehy (FMTE), Sud-est de la Côte d'Ivoire
}

\author{
Joël Emmanuel N'Gouan ABROU ${ }^{1 *}$, Djaha KOUAMÉ ${ }^{2}$ et Constant Yves ADOU YAO ${ }^{1,3}$ \\ ${ }^{1}$ Université Félix Houphouët-Boigny, UFR Biosciences, 22 BP 582 Abidjan 22, Côte d'Ivoire. \\ ${ }^{2}$ Université Jean Lorougnon Guédé, UFR environnement, BP 150 Daloa, Côte d'Ivoire. \\ ${ }^{3}$ Centre Suisse de Recherches Scientifiques (CSRS), 01 BP 1303, Abidjan Côte d'Ivoire. \\ *Auteur pour correspondance, E-mail: ngouanemmanueljoel@gmail.com; Tel: (+225) 47049517 ,
}

$$
\text { (+225) } 43672573
$$

\section{REMERCIEMENTS}

La présente étude s'inscrit dans le cadre d'un processus de conservation, de protection et de préservation de la FMTE piloté par l'organisme International Foundation for Science (IFS).

\section{RÉSUMÉ}

La Forêt des Marais Tanoé-Ehy (FMTE) a été identifiée comme étant un site hautement prioritaire pour la conservation des primates en Afrique de l'Ouest. Cependant, ce site est soumis aux pressions anthropiques, engendrant les modifications dans la flore et la structure des communautés végétales. Cette étude a eu pour objectifs d'identifier les communautés végétales dans l'espace de la FMTE et de déterminer leurs caractéristiques floristiques et structurales. Les données ont été collectées dans différentes unités d'occupations du sol et à travers une Analyse Canonique de Correspondances (ACC), des groupes de relevés ont été obtenus ainsi que les espèces indicatrices des différentes formations végétales. Au total, 568 espèces végétales ont été inventoriées. Ces espèces se répartissent entre cinq types de végétations parmi lesquelles les forêts sur terre ferme sont les plus diversifiées. Dans les habitats plus stables de l'intérieur du site, les espèces indicatrices identifiées peuvent constituer des indicateurs clés pour le suivi environnemental.

(C) 2019 International Formulae Group. All rights reserved

Mots clés : zone humide,forêt marécageuse, espèces indicatrices, pressions anthropiques, Système agroforestier.

\section{Floristic diversity of plant communities in the space of the Tanoe-Ehy swamp forest, southeast of Côte d'Ivoire}

\begin{abstract}
The Tanoé-Ehy Marsh Forest (FMTE) has been identified as a high priority site for the conservation of primates in West Africa. However, this site is subject to anthropogenic pressures, which created modifications in flora composition and structure. The purpose of this study was to identify plant communities in the FMTE area and to determine their floristic and structural characteristics. The data were collected in different landuse units and through a Canonic Correspondence Analysis (CCA), groups of surveys were obtained as well as indicator species of the different plant formations. A total of 568 plant species have been inventoried. These
\end{abstract}


species are divided into five types of vegetation, of which the mainland forests are the most diversified. In more stable habitats within the site, identified indicator species may be key indicators for environmental monitoring.

(C) 2019 International Formulae Group. All rights reserved

Keywords: Wetland; Swamp forest; Indicator species, Agroforestry system; Anthropogenic pressures.

\section{INTRODUCTION}

Les zones humides sont des habitats intermédiaires entre les milieux terrestres et aquatiques. Elles se caractérisent par une nappe phréatique proche de la surface et par une forte productivité biologique (Barnaud et Fustec, 2007). Ces écosystèmes couvrent $9 \%$ de la superficie mondiale des terres (Zedler et Kercher, 2005) et fournissent divers services écologiques tels que l'amélioration de la qualité de l'eau, la régularisation des débits des cours d'eau, la séquestration du carbone et constituent un support de la biodiversité (Levison et al., 2013).

Les superficies mondiales des zones humides ont cependant, regressé de 50\% au cours des derniers siècles et la plupart de celles qui existent encore sont dégradées (Egnankou, 2015). Cette dégradation est due principalement à l'urbanisation, l'agriculture et l'exploitation forestière (Marabushu, 2011). Ces transformations (perte et dégradation d'habitat) affectent la structure des paysages aux quels s'ajoutent de nombreuses conséquences écologiques (Burel et Baudry, 2001).

En Côte d'Ivoire, les zones humides côtières et continentales représentent $2 \%$ du territoire national (Egnankou, 2015). Ces écosystèmes jouent un rôle essentiel dans la subsistance des communautés rurales et la protection des sols et des eaux (Hussain et al., 2007). Ces zones humides fournissent des biens précieux et rendent de nombreux services : épuration de l'eau, atténuation des crues, soutien d'étiage. Ce sont des zones de recharge et de protection des nappes d'eau souterraines, de maintien des stocks et de la qualité des eaux domestiques, agricole et industrielle, de piégeage des sédiments et des polluants et de productions biologiques. De ce fait, la Côte d'Ivoire a ratifié la convention de Ramsar et a inscrit 6 sites sur la liste des zones humides d'importance internationale que sont le Parc national d'Azagny, le complexe Sassandra - Dagbégo, Fresco, la zone humide de Grand-Bassam, les Iles Ehotilés - Essouman et la forêt Classée de $\mathrm{N}^{\prime} G a n d a-N^{`} G a n d a$. Malgré tout, ces zones humides sont menacées de disparition car elles ne reçoivent ni l'intérêt, ni la protection qu'elles méritent et sont soumises à de rudes épreuves. Par exemple, dans le Sud-Est de la Côte d'Ivoire, la baisse des écoulements des eaux continentales est due à des actions anthropiques notammentla déforestation généralisée, la pêche intensive et l'utilisation incontrôlée d'intrants chimiques agricoles. Ces actions provoquent des modifications physico-chimiques et une eutrophisation des eaux dans ces zones très fragiles, entraînant l'implosion des espèces exotiques et la disparition de ressources biologiques d'importance capitale (Egnankou, 2015). Ainsi, la reconversion de ces milieux humides en zones agricoles conduit à des dysfonctionnements hydrauliques tels que l'aggravation des crues et des pollutions, les sécheresses, les inondations, l'érosion des côtes, la baisse des captures de poissons et la diminution de la diversité biologique. De nombreux habitats, supports d'une grande richesse écologique, deviennent de plus en plus vulnérables.

La Forêt des Marais Tanoé-Ehy (FMTE), site de la présente étude, a été identifiée comme étant hautement prioritaire pour la conservation des primates (Koné et al., 2008). Il s'agit d'une zone humide dont le processus de classement en Reserve Naturelle Volontaire (RNV) a débuté en 2006. Elle est, en effet, l'un des derniers vestiges de ce type de forêt. En attendant son classement, la FMTE est soumise à de nombreuses pressions de la part des populations riveraines : mise en place de nombreuses de cultures de rente (Palmeraie, cacaoyères...); ce qui grignote ses superficies. Elle est également soumise 
aux activités de pêche pendant presque toute l'année. Toutes ces menaces risquent de nuire à la pérennisation des habitats qui composent le site de la FMTE. La modification des composantes de ces écosystèmes, et notamment celle de leurs communautés floristiques, peut constituer une menace sérieuse au maintien de leurs fonctions écosystémiques. Pourtant, en ce qui concerne la FMTE et des sites situés à sa périphérie, ces habitats sont encore mal connus. Des études réalisées par Abrou et al. (2017) et des prospections dans ce milieu ont révélé une multitude d'habitats dans la FMTE. Il s'agit entre autre des forêts marécageuses, des forêts sur sol à inondation temporaire, des forêts sur terre ferme. Ailleurs, il est démontré que des espèces à niche écologique particulière sont inféodées à de tels habitats (Gnagbo et al., 2015). Aussi, la disparition de certaines espèces exclusives et leur remplacement par des espèces généralistes dans des milieux particuliers, diminuent le taux d'accumulation de la matière organique et donc celui du carbone (Moore et Coste, 2006). Or, l'espace de la FMTE, comme les alentours de la plupart des aires protégées de la Côte d'Ivoire (Sangne et al., 2015) est sujet à diverses activités et pratiques des populations riveraines dont les effets non négligeables ne sont pas connus. D'où notre présente étude qui a pour objectif général de caractériser les différents habitats de la FMTE ainsi que les biotopes dans ses environs immédiats. Spécifiquement, l'étude a consisté à identifier les communautés végétales sur la base de paramètres mésologiques et déterminer des caractéristiques floristiques et structurales des habitats dans l'espace de la Forêt des Marais Tanoé-Ehy.

\section{MATERIEL ET METHODES \\ Site d'étude}

La FMTE, située au Sud-est de la Côte d'Ivoire, s'étend sur une superficie de 12000 ha et est limitée à l'Ouest par la lagune Ehy, du Sud à l'Est par le fleuve Tanoé, et au Nord par des terroirs villageois notamment Yaoakakro et Kongodjan. Elle est à cheval sur les Sous-préfectures de Noé, Nouamou et Tiapoum (Figure 1).
Le site bénéficie d'un climat de type sub-équatorial à quatre saisons dont deux saisons sèches (une petite saison sèche pendant le mois d'août et une grande saison sèche de décembre à février) et deux saisons pluvieuses (une grande saison pluvieuse de mars à juillet, avec le maximum de précipitation en juin et une petite saison pluvieuse d'octobre à novembre) selon Adou Yao (2007). Les précipitations moyennes annuelles sont comprises entre $1400 \mathrm{~mm}$ et $1600 \mathrm{~mm}$ (Brou et al., 2005). La température varie entre $22^{\circ} \mathrm{C}$ et $30^{\circ} \mathrm{C}$ avec une moyenne de $26^{\circ} \mathrm{C}$. En ce qui concerne la végétation, la FMTE est située dans le secteur littoral, caractérisé par la présence des forêts marécageuses (Guillaumet et Adjanohoun, 1971). Elle est couverte en grande partie d'une végétation de forêt sempervirente, marécageuse sur un sol hydromorphe (Guillaumet et Adjanohoun, 1971). Les autres formations végétales observées sont la raphiale, la forêt inondable et la forêt de terre ferme (Adou Yao, 2007).

L'économie de la zone est dominée par l'agriculture; ce qui a eu pour conséquence le remplacement des écosystèmes forestiers par de vastes plantations de cacaoyers et de palmiers à huile, à la périphérie de la FMTE, appartenant aux villageois et à la Palmeraie Industrielle de Côte d'Ivoire (PALMCI). À ces plantations, s'ajoute une unité agroindustrielle spécialisée dans la production d'huile de palme (Zadou et al., 2011). Les zones de cultures se rencontrent à la périphérie de la FMTE, où la pression anthropique est très forte. À l'intérieur de la FMTE, certaines zones, heureusement de petites surfaces, sont exploitées. Quatre principales cultures pérennes que sont le palmier à huile, le cocotier, le cacaoyer et l'hévéa sont exploitées. Les cultures vivrières sont généralement représentées par le manioc et la banane plantain. Ces cultures sont fortement entremêlées, et avec des jachères de différents âges qu'on rencontre généralement à la périphérie de la FMTE. Le relief est caractérisé par une frange littorale très peu élevée. Les altitudes sont au maximum de 10 à $12 \mathrm{~m}$. 


\section{Collecte de données}

Plusieurs types d'habitats sont présents dans l'espace de la FMTE: forêts denses inondables (forêt marécageuse et forêt temporairement inondée), forêts sur terre ferme, jachères dont l'âge varie de 2 ans (jachère jeune) à 17 ans (jachère ancienne) et des plantations de cacaoyer et de palmiers (Abrou et al., 2017). Pour prendre en compte cette hétérogénéité du milieu, les inventaires botaniques ont été réalisés dans chacun de ces habitats. Ainsi des placettes de $400 \mathrm{~m}^{2}(20 \mathrm{~m}$ x $20 \mathrm{~m}$ ) ont été mises en place. Au total, 134 placettes ont été réalisées (Figure 1) : 41 placettes dans les forêts marécageuses, 25 placettes dans les forêts inondées temporairement, 26 placettes dans les forêts sur terre ferme, 21 placettes dans les jachères (10 dans les jeunes jachères et 11 dans les vieilles jachères) et 21 placettes dans les plantations (10 dans les cacaoyères et 11 dans les palmeraies). La majorité des parcelles situées hors de la forêt se trouvaient à l'Ouest de la réserve car les autres bordures faisaient frontière avec le fleuve Tanoé (Figure 1).

À l'intérieur des placettes, tous les individus arborescents dont le diamètre à hauteur de poitrine (dhp) est supérieur ou égal à $2,5 \mathrm{~cm}$, ont été dénombrés. Les espèces auxquelles appartiennent ces individus ont été identifiées. La présence des espèces des autres types morphologiques (lianes et herbes) et les arborescents dont le diamètre à hauteur de poitrine (dhp) est inférieur à $2,5 \mathrm{~cm}$ a été notée. Les noms des espèces ont été mis à jour à partir de Lebrun et Stork (1991-1997) utilisée par plusieurs botanistes (Aké-Assi, 2001 ; Aké-Assi, 2002). La nomenclature des espèces, suivie dans cette étude, est celle de APG IV (2016). Des paramètres mésologiques ont été pris en compte. Ces paramètres concernent le niveau de dégradation de la parcelle, la présence de l'eau (degré d'inondation du sol), le type de végétation, la texture du sol et la topographie. Une échelle de dégradation allant de 0 à 5 (Tiébré et al., 2016a) a été choisie pour mettre en évidence les niveaux de perturbation de la végétation (Tableau 1). Les critères permettant de mesurer cette dégradation sont les traces de coupes de bois et de lianes, les traces de chasse ou pièges et les traces de feux, jachères et champs ou plantations. La perturbation est dite nulle lorsqu'aucune trace humaine n'est observée. Elle est de plus grande lorsque plusieurs indices de dégradation sont présents dans l'habitat. Le degré d'inondation est apprécié visuellement sur toute pratiquement toute l'année (période pluvieuse et sèche). Il s'est agi de préciser si la végétation est sur une terre ferme, inondée périodiquement ou inondé en permanence. Le type de végétation (jachère, plantation, ou forêt) a été précisé. La texture du sol (argileux, sableux ou limoneux) a été notée à partir de la méthode du toucher (Baize et Jabiol, 1995). Pour ce faire, on prend une poignée de sol interessé sec ou humide, ensuite on la presse fortement, on peut aussi la frotter entre le pouce et les autres doigts de la main (Tableau 2). Enfin, pour la topographie, il a été vérifié si le biotope était en bas de pente, à mi- pente ou en haut de pente (sommet).

\section{Analyse des données}

La richesse floristique de l'espace FMTE a été déterminée en mettant en exergue le nombre d'espèces, le nombre de genres et de familles (Aké-Assi, 2002). Ce dénombrement a permis d'avoir une idée globale sur la diversité des espèces du site et de ses environs immédiats.

Les données floristiques relatives aux espèces arborescentes $(\mathrm{dhp} \geq 2,5 \mathrm{~cm})$ et mésologiques ont été ensuite soumises à une Analyse Canonique des Correspondances (ACC) avec le logiciel CANOCO (Canonical Community Ordination). Cette analyse a permis de caractériser les groupes de relevés floristiques en relation avec les paramètres mésologiques dans l'espace FMTE. Cette analyse a aussi permis de déterminer les facteurs environnementaux responsables de la présence et de l'abondance des espèces floristiques. Pour chaque groupe de relevés, des espèces indicatrices des formations végétales ont été identifiées à travers la méthode IndVal (Dufrêne, 2004) à travers la formule suivante :

$\operatorname{IndVal}_{\mathrm{ij}}=\mathrm{A}_{\mathrm{ij}} \times \mathrm{B}_{\mathrm{ij}} \times 100$ 
Avec IndVal $\mathrm{ij}_{\mathrm{ij}}$ : Valeur indicatrice de l'espèce i pour le groupe $\mathrm{j} ; \mathrm{A}_{\mathrm{ij}}=$ Nindividusij / Nindividusi et $\mathrm{B}_{\mathrm{ij}}=\mathrm{Nsites}_{\mathrm{ij}} / \mathrm{Nsites}_{\mathrm{j}}$; Nind $_{\mathrm{ij}}$ : nombre moyen d'individu d'espèce $\mathrm{i}$ dans le groupe $\mathrm{j}$; Nindi : nombre moyen d'individu d'espèce $\mathrm{i}$; Nsites $_{\mathrm{ij}}$ : nombre de sites du groupe $\mathrm{j}$ où l'espèce $\mathrm{i}$ est présente ; Nsites $_{\mathrm{j}}$ : nombre de sites du groupe $j$.

Cette methode IndVal tient compte de l'abondance, de la spécificité et de la fidélité des espèces dans les différents relevés. Chaque groupe de relevés a été caractérisé par les deux espèces présentant les plus forte Valeur Indicatrices (IV). La diversité spécifique des groupes de relevés a été estimée par le nombre d'espèces. Le genre et la famille ont été attribués pour chacune des espèces, suivant la classification APG IV (APG IV, 2016). Aussi, des indices de diversité ont été calculés noramment l'indice de Shannon (H) qui a permis d'évaluer la diversité de chaque groupe de rélevé à partir de la formule mathématique suivante :

$$
\mathrm{H}^{\prime}=-\Sigma(\mathrm{Ni} / \mathrm{N}) \ln (\mathrm{Ni} / \mathrm{N})
$$

Dans cette formule, H' représente l'indice de diversité de Shannon, Ni est le nombre d'individus d'une espèce $\mathrm{i}$ et $\mathrm{N}$ le nombre total d'individus de toutes les espèces.
Nous avons calculé l'indice d'équitabilité de Piélou (E) qui a permis de dire si le milieu écologique considéré ou l'habitat est dominé par une espèce. Autrement dit, la valeur de cet indice tend vers 0 lorsqu'une ou très peu d'espèces dominent largement et elle est égale à 1 lorsque toutes les espèces ont la même abondance. La formule est la suivante :

$$
\mathrm{E}=\mathrm{H}^{\prime} / \ln \mathrm{S}
$$

où $\mathrm{E}$ désigne l'indice d'équitabilité de Piélou, H' est l'indice de Shannon et $\mathrm{S}$ représente le nombre total d'espèces de la parcelle ou de l'espace concerné

Enfin, la densité et l'aire basale de chaque groupe relévé ont été déterminées. Des comparaisons de moyennes ont été faites à l'aide des tests de Kruskas wallis et de posthoc de Dunn avec le logiciel R-3.2.0. extension « Biodiversity ».

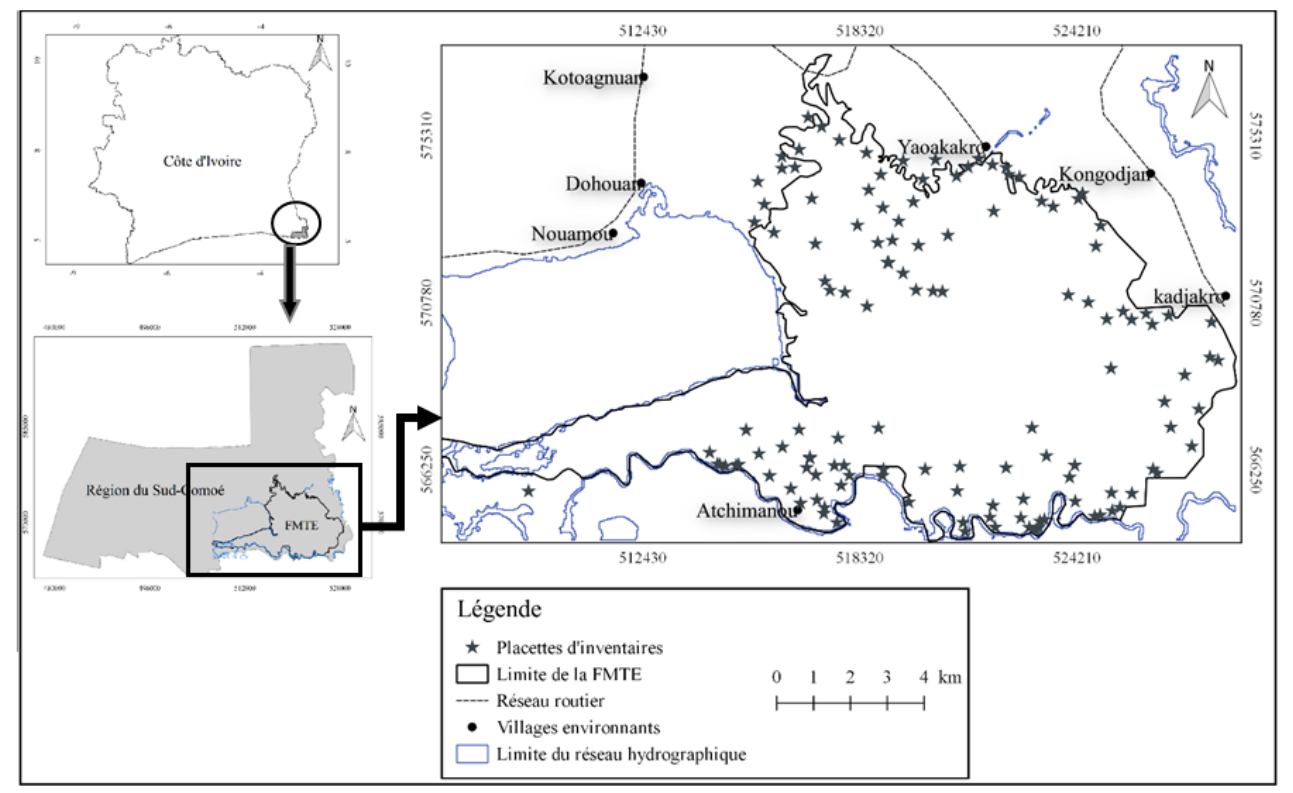

Figure 1 : Cartes de localisation de la Forêt des Marais Tanoé-Ehy et des parcelles d'inventaire. 
Tableau 1: Différentes classes de dégradation de la végétation en fonction de l'intensité des activités humaines.

\begin{tabular}{ll}
\hline Observations & Niveau de dégradation \\
\hline Aucune présence anthropique & 0 \\
\hline Coupes de bois et / ou de lianes dans la forêt & 1 \\
\hline Traces de chasse (douilles) ou pièges dans la forêt & 1 \\
\hline Traces de feux ou zone de fabrication de charbon dans la forêt & 2 \\
\hline Pistes dans la forêt & 1 \\
\hline Jachères (jeunes) & 3 \\
\hline Strate herbacée uniquement après agriculture & 3 \\
\hline Champs ou plantations & 5 \\
\hline
\end{tabular}

Tableau 2 : Récapitulatif des différents indices de la méthode du toucher.

\begin{tabular}{lll}
\hline \multirow{2}{*}{ Test } & Résultat & Interpretation \\
\hline \multirow{2}{*}{ Toucher de la terre seche } & Soyeux ou talqueux & Abonndance de limons fins \\
\cline { 2 - 3 } & Savonneux & $\begin{array}{l}\text { Abondance de limons } \\
\text { grossiers }\end{array}$ \\
\cline { 2 - 3 } & rigueux & Sable grossier \\
\hline \multirow{2}{*}{$\begin{array}{l}\text { Réalisation d'un boudin de } \\
\text { terre humide }\end{array}$} & Possible & Arigile $>10 \%$ \\
\cline { 2 - 3 } & Impossible & Argile $<10 \%$ \\
\hline \multirow{3}{*}{$\begin{array}{l}\text { Réalisation d'un anneau } \\
\text { avec le boudin de terre }\end{array}$} & $\begin{array}{l}\text { Fissuration avant } \\
\text { ouverture de l'anneau }\end{array}$ & $\begin{array}{l}\text { Limon }>\text { argile; } \\
\text { Argile }<30 \%\end{array}$ \\
\cline { 2 - 3 } & Fermeture & $\begin{array}{l}\text { Limon }<\text { argile; } \\
\text { Argile }<30 \%\end{array}$ \\
\cline { 2 - 3 } & Anneau réalisable & Arigile $>30 \%$ \\
\hline
\end{tabular}

\section{RESULTATS}

\section{Richesse floristique globale}

L'ensemble des habitats de la FMTE et ses environs immédiats, est riche de 568 espèces végétales reparties en 352 genres et 100 familles. Ces espèces sont constituées en 343 espèces arborescentes $(60 \%), 165$ espèces lianescentes $(29 \%)$ et 60 espèces herbacées (11\%). Les genres les plus représentés sont Ficus (10 espèces), Cola (9 espèces), Ouratea et Salacia (8 espèces chacun), Combretum, Memecylon, Trichilia, et Xylopia (6 espèces chacun). Aussi $42,60 \%$ des genres sont représentés par une seule espèce. Les familles les plus dominantes en espèces sont les Fabaceae (58 espèces, soit 10,2\%), les Rubiaceae (55 espèces, soit $9,7 \%)$, Apocynaceae (29 espèces, soit 5,1\%). Les valeurs sont consignées dans le tableau 3.

\section{Identification des groupes de relevés}

Parmi les espèces recensées dans cette étude, 343 espèces arborescentes(dont le diamètre à hauteur de poitrine (dhp) est 
supérieur ou égal à $2,5 \mathrm{~cm}$ ) ont été soumises à une analyse Canonique de Correspondances.

Ainsi, les deux premiers axes de l'Analyse Canonique de Correspondance (ACC) expliquent $74,8 \%$ de la variance totale (Figure 2). Cinq (05) groupes de relevés ont été identifiés. Le groupe 1 (G1) est composé uniquement des relevés effectués au sein des forêts marécageuses. Ces relevés sont des parcelles sur sol constamment inondé et situées dans les bas-fonds. Le groupe 2 (G2) est constitué de tous les relevés effectués dans les forêts sur sol temporairement inondé. Les facteurs favorables à la présence et à l'abondance des espèces dans ces relevés, sont généralement des plaines d'inondations présentant une faible pente. On les retrouve pour la plupart dans les zones de mi- pente. Le groupe 3 (G3) est celui des parcelles issues des forêts sur terre ferme. Ces parcelles ont un degré de dégradation allant de 1 (Ecorçage) à 3 (coupe de bois). Elles sont aussi caractérisées par des sols exondés. Le groupe 4 (G4) est formé de $73,9 \%$ des parcelles de jachères quel que soit l'âge. Dans ces parcelles, les facteurs qui conditionnent la présence et l'abondance des espèces sont les perturbations humaines et le type de formation végétale. Enfin, le groupe 5 (G5) renferme l'ensemble des parcelles issues de plantations cacaoyères et des palmeraies.

\section{Caractéristiques floristiques et structurales des groupes de relevés}

$\mathrm{Au}$ niveau des biotopes forêts, les espèces indicatrices des différents milieux sont différentes selon que l'on soit dans des marécages, des habitats temporairement inondés ou sur terre ferme (Tableau 4). Dans les relevés de forêts marécageuses (G1), les espèces caractéristiques sont Hallea ledermannii $(\mathrm{IV}=68,12)$ et Xylopia rubescens $(\mathrm{IV}=57,32)$. Dans les parcelles de forêts temporairement inondées (G2), Uapaca paludosa (IV $=87,34)$ et Symphonia globulifera (IV $=84,29)$ sont les espèces indicatrices.

Les parcelles de forêts sur terre ferme (G3), ont comme espèces indicatrices,
Tetrorchidium didymostemon $(\mathrm{IV}=62,83)$ et Funtumia africana (IV = 47,98).

Les espèces indicatrices des parcelles soumises aux fortes activités humaines sont différentes de celles identifiées pour les forêts où l'acivité anthropique est moins accentuée (Tableau 4). Dans les relevés de jachères (G4) on rencontre préférentiellement Myrianthus arboreus et Albizia ferruginea (IV $=66,67$ chacune). Dans les parcelles cacaoyères et aux palmeraies (G5), les espèces indicatrices des milieux fortement pertubés sont : Cola nitida (IV $=70$ ) et Rauvolfia vomitoria (IV = 63,53).

Les cinq groupes de relevés identifiés dans l'espace FMTE présentent une richesse moyenne de la placette $\left(499 \mathrm{~m}^{2}\right)$ qui varie de 9,52 à 51,18 espèces (Tableau 4). En tenant compte des zones anthropisées, les jachères (G4) ont une valeur moyenne plus élevée $(23,47 \pm 10,85$ espèces $)$ par rapport aux plantations (9,52 \pm 4,73 espèces). Si l'on considère les habitats moins anthropisés (biotopes forestiers), les forêts sur terre ferme (G3) sont les plus riches avec une valeur moyenne de 51,18 $\pm 20,39$ espèces. Elles sont suivies des forêts sur sol temporairement inondé (G2) avec une valeur moyenne de $25,68 \pm 13,17$ espèces. Les forêts marécageuses (G1) sont les moins riches avec un nombre moyen de 11,45 $\pm 5,87$ espèces. Les différences entre les moyennes sont significatives (Test de Kruskal Wallis : $\chi^{2}=$ $83,34 ; p<0,0001)$.

Les indices de diversité varient d'un groupe de relevé à l'autre (Tableau 4). Pour ce qui est de l'indice de diversité de Shannon $\left(\mathrm{H}^{\prime}\right)$, la moyenne est comprise entre 0,74 et 3,27 . Les jachères (G4) sont plus diversifiées avec une valeur moyenne de 2,66 $\pm 0,5$ comparativement aux plantations $\left(\mathrm{H}^{\prime}=0,74 \pm\right.$ $0,5)$. En considérant les habitats moins anthropisés, les forêts sur terre ferme (G3) sont les plus diversifiées avec une valeur moyenne de 3,27 $\pm 0,47$. Elles sont suivies des forêts sur sol temporairement inondé $\left(\mathrm{H}^{\prime}=\right.$ $2,54 \pm 0,52)$. Les forêts marécageuses $(\mathrm{G} 1)$ sont les moins diversifiées $\left(\mathrm{H}^{\prime}=1,73 \pm 0,56\right)$. Les différences entre les moyennes sont significatives (Test de Kruskal Wallis : $\chi^{2}=$ 
93,23; $p<0,0001)$. Les mêmes tendances sont observées pour l'indice d'équitabilité de Piélou (Tableau 4).

En ce qui concerne paramètres structuraux, les plus fortes densités de tiges ont été rencontrées dans les forêts sur terre ferme (2697,11 tiges/ha). Ce type de forêt est suivi des jachères (1227,97 tiges/ha) et les forêts marécageuses (1069,51 tiges/ha). Les plantations sont les moins denses (Tableau 3). À l'exception des forêts marécageuses, les aires basales (41,68 à 49,24 m²/ha) des autres types de forêt sont plus élevées que celles des espaces anthropisés (Tableau 4). L'aire basale dans les plantations est la plus faible $(9,84$ $\mathrm{m}^{2} / \mathrm{ha}$ ).
Le graphe de distribution des tiges par classe de diamètre ont donné deux formes différentes (Figure 3). Dans les groupes G1, G2et G3 qui sont tous issus du biotope forêt, les tiges de petits diamètres [2,5-10[ sont les plus nombreuses. Elles sont suivies des tiges dont les diamètres sont compris entre 10 et 20 $\mathrm{cm}$, puis de celles ayant des diamètres supérieurs. On constate une évolution régressive des tiges, des plus petits diamètres vers les plus grands, donnant à la courbe une allure en « $\mathbf{J}$ inversé ». Les autres groupes issus des jachères et des plantations ont présenté une courbe dont l'allure est en forme de dent de scie.

Tableau 3 : Liste des familles les plus abondantes en espèces.

\begin{tabular}{lll}
\hline Familles & Nombre d'espèces & Pourcentage (\%) \\
\hline Fabaceae & 58 & 10,2 \\
\hline Rubiaceae & 55 & 9,7 \\
\hline Apocynaceae & 29 & 5,1 \\
\hline Annonaceae & 25 & 4,4 \\
\hline Malvaceae & 20 & 3,5 \\
\hline Moraceae & 20 & 3,5 \\
\hline Arecaceae & 19 & 3,3 \\
\hline Phyllanthaceae & 18 & 3,2 \\
\hline Sapindaceae & 15 & 2,6 \\
\hline Euphorbiaceae & 14 & 2,5 \\
\hline Autres familles & 295 & 51,9 \\
\hline
\end{tabular}




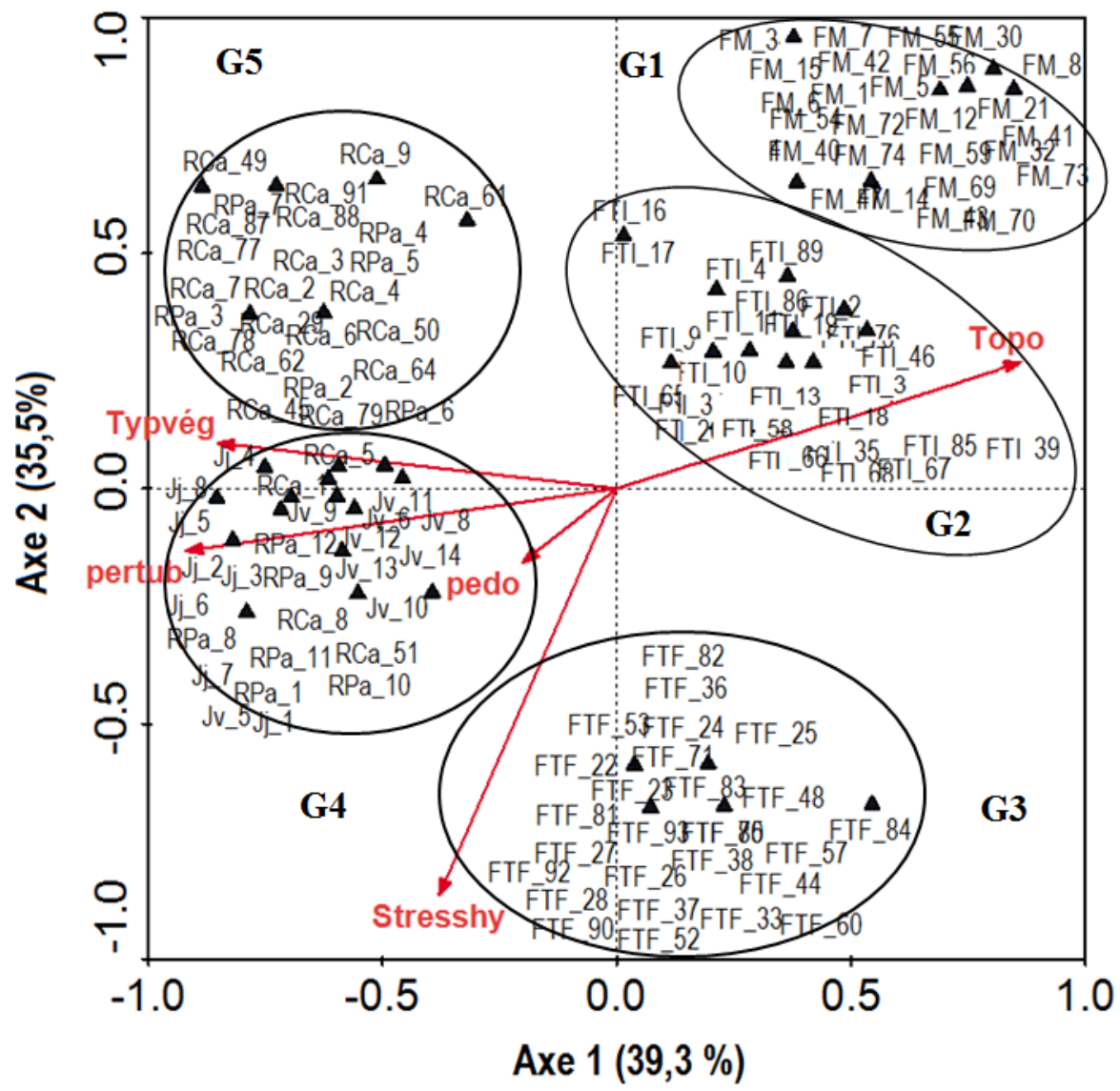

Figure 2: Ordination des parcelles par l'Analyse Canonique de Correspondance (ACC).

FM : Parcelles de Forêt marécageuse ; FTI : Parcelles de Forêt temporairement inondé ; FTF : Parcelles de Forêt sur terre ferme ; Jv : Parcelles de Vieille jachère ; Jj : Parcelles de Jeune jachère ; RCa : Parcelles de plantations de cacaoyer ; RPa : Parcelles de palmeraies; Topo: Topographie; Stresshy: Stress hydrique, Pedo: Texture du sol ; Pertub : Perturbation humaine ; Typvég: Type de formation végétale ; position des rélevés

Tableau 4 : Caractéristiques floristiques et structurales des différents groupes de relevés.

\begin{tabular}{|c|c|c|c|c|c|c|c|}
\hline $\begin{array}{l}\text { Groupes } \\
\text { de relevés }\end{array}$ & $\begin{array}{l}\text { Espèces } \\
\text { caractéristiques }\end{array}$ & IV & Espèces & Shannon & Equitabilité & $\begin{array}{l}\text { Densité } \\
\text { (Tiges/ha) }\end{array}$ & $\begin{array}{l}\text { Aire basale } \\
\left(\mathrm{m}^{2} / \mathrm{ha}\right)\end{array}$ \\
\hline \multirow{2}{*}{ G1 } & Hallea ledermannii & 68,12 & \multirow{2}{*}{$\begin{array}{l}11,45 \quad \pm \\
5,87^{\text {a }}\end{array}$} & \multirow{2}{*}{$1,73 \pm 0,56^{\mathrm{b}}$} & \multirow{2}{*}{$0,74 \pm 0,14^{\mathrm{b}}$} & \multirow{2}{*}{1069,51} & \multirow{2}{*}{25,85} \\
\hline & Xylopia rubescens & 57,32 & & & & & \\
\hline \multirow[b]{2}{*}{ G2 } & Uapaca paludosa & 87,34 & \multirow{2}{*}{$\begin{array}{l}25,68 \pm \\
13,17^{\mathrm{b}}\end{array}$} & & \multirow{2}{*}{ $\pm 0,80 \pm$} & \multirow[b]{2}{*}{1818} & \multirow[b]{2}{*}{41,68} \\
\hline & Symphonia & 8 & & $0,52^{\mathrm{c}}$ & & & \\
\hline \multirow{3}{*}{ G3 } & Tetrorchidium & & \multirow{3}{*}{$\begin{array}{l}51,18 \pm \\
20,39^{\mathrm{c}}\end{array}$} & \multirow{3}{*}{$3,27 \pm 0,47^{\mathrm{d}}$} & \multirow{3}{*}{$0,85 \pm 0,06^{\mathrm{cd}}$} & \multirow{3}{*}{2697,11} & \multirow{3}{*}{49,24} \\
\hline & didystemon & 62,83 & & & & & \\
\hline & Funtumia africana & 47,98 & & & & & \\
\hline \multirow{2}{*}{ G4 } & Albizia ferruginea & 66,67 & \multirow{2}{*}{$\begin{array}{l}23,47 \pm \\
10,85^{\mathrm{b}}\end{array}$} & \multirow{2}{*}{$2,66 \pm 0,5^{\mathrm{c}}$} & \multirow{2}{*}{$0,88 \pm 0,07^{\mathrm{d}}$} & \multirow{2}{*}{1227,97} & \multirow{2}{*}{31,66} \\
\hline & Myrianthus arboreus & 66,67 & & & & & \\
\hline \multirow{2}{*}{ G5 } & Cola nitida & 70 & $9,52 \pm$ & \multirow{2}{*}{$0,74 \pm 0,5^{\mathrm{a}}$} & \multirow{2}{*}{$0,34 \pm 0,16^{\mathrm{a}}$} & \multirow{2}{*}{144,04} & \multirow{2}{*}{9,84} \\
\hline & Rauvolfia vomitoria & 63,53 & $4,73^{\mathrm{a}}$ & & & & \\
\hline
\end{tabular}

Pour une même colonne, les valeurs moyennes affectées de la même lettre ne sont pas significativement différentes au seuil de $5 \%$. 


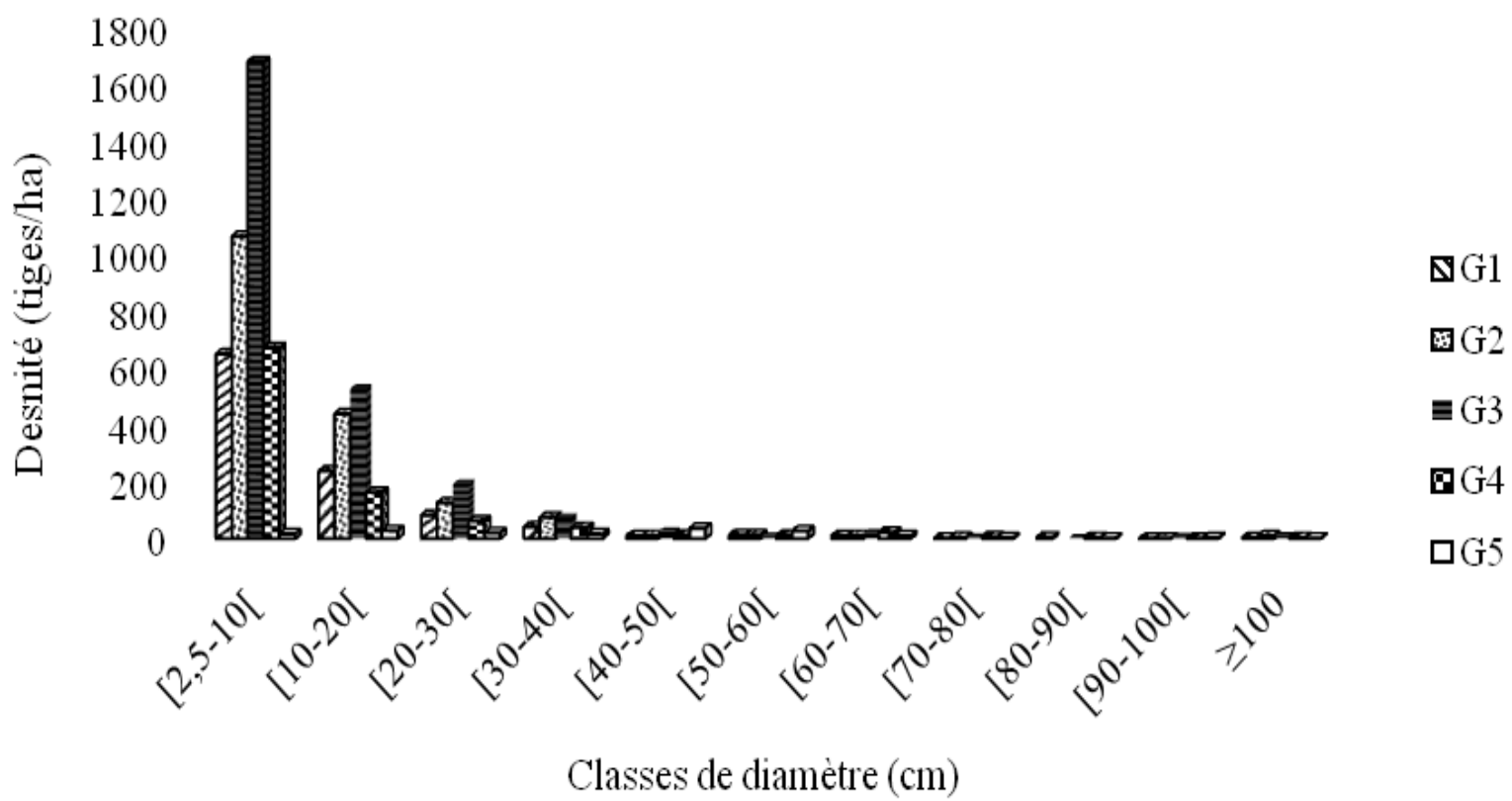

Figure 3 : Structure démographique en classes de diamètre des différents groupes de relevés.

\section{DISCUSSION}

La présente étude avait pour objectif de déterminer des caractéristiques floristiques et structurales des habitats dans l'espace de la Forêt des Marais Tanoé-Ehy. Elle a aussi permettre de mettre en évidence des paramètres mésologiques pouvant être responsables de la présence et de l'abondance des espèces. Tout d'abord l'étude a montré que $14,98 \%$ de la richesse floristique de la Côte d'Ivoire se rencontre dans l'espace de la FMTE, selon les estimations de (Aké-Assi, 2001). Ce taux reste supérieur à celui de Tiébré et al. (2016b) qui ont obtenu une proportion de $09,5 \%$. Les familles botaniques les plus dominantes sont les Fabaceae, les Rubiaceae et les Apocynaceae. L'abondance de ces familles s'expliquerait par le fait que la FMTE se situe dans la région forestière Guinéo-Congolaise qui est le domaine de prédilection des Rubiaceae (Aké-Assi, 2002). Des auteurs comme Missa et al. (2015) dans les zones périphériques de la FMTE et Nshimba et al. (2005) dans la forêt marécageuse de l'île Mbiye de la République Démocratique du Congo ont fait des observations similaires.
L'Analyse Canonique de Correspondances (ACC) a permis de distinguer cinq groupes de relevés sur la base de cinq (05) variables explicatives: la topographie, le stress hydrique, la texture du sol, la perturbation humaine et le type de formation végétale. A travers cette analyse, des biotopes situés dans les bas-fonds dont le sol est constamment ou périodiquement inondé avec moins d'agressions anthropiques, sont séparés des biotopes de haut de pente sur terre ferme et qui subissent régulièrement des pressions de la part des populations riveraines. Cette séparation traduit les faibles activités anthropiques dans des espaces inondés et dans les zones marécageuses. Il s'agit en effet d'habitats très difficiles d'accès et leur instabilité serait peu favorable aux pratiques culturales des populations locales. La typologie des groupes de relevés est donc imputable aux variables environnementales notamment la topographie et l'humidité du sol. En général pour les régions tropicales où la variation altitudinale est faible comme c'est le cas pour la FMTE, c'est la topographie qui est couramment citée comme variable discriminante à laquelle peut être corrélé le type de sol ou l'humidité du sol (Wala, 2004). 
Ainsi, les parcelles issues des forêts où le sol est constamment ou temporairement inondé sont celles qui sont situées généralement dans les bas-fonds. Ce sont des parcelles dont les conditions écologiques qui prévalent sont difficiles (Adou Yao, 2007), d'où la présence de rares espèces qui peuvent s'accommoder à ce type d'habitat. On retrouve dans ces habitats des espèces telles que Hallea ledermannii, Uapaca paludosa, Raphia hoockeri et Xylopia rubescens. En effet, dans ces milieux marécageux, la plupart des espèces développent des systèmes racinaires qui pourront leur permettre de survivre. C'est le cas par exemple de Xylopia rubescens avec des racines échasses et de Raphia hookeri qui développent des pneumatophores pour se maintenir dans ces milieux. Ces milieux marécageux ne permettent donc que l'établissement d'un faible nombre d'espèces adaptées aux conditions d'hydromorphie permanente. Ainsi, les forêts marécageuses sont les moins riches et les moins diversifiées. Selon Adou Yao (2007), les milieux marécageux sont en général, les moins riches en espèces, du fait des conditions extrêmes. Par contre, le retrait temporaire de l'eau pourrait être favorable aux espèces végétales généralistes (Baphia nitida, Heisteria parvifolia, Carapa procera...) d'où l'augmentation de la richesse dans les forêts périodiquement inondées.

Sur les terres exondées (forêt sur terre ferme), au sein de la FMTE, la richesse et la diversité des espèces augmentent une fois encore par rapport à celles obtenues dans les forêts marécageuses et les forêts périodiquement inondées. Les parcelles des forêts sur terre ferme ont pour espèces indicatrices Tetrorchidium didymostemon et Funtumia africana qui sont des espèces héliophiles. En effet, ces habitats de terre ferme sont situés pour la plupart à la lisière de la forêt. Il s'agit donc des habitats qui forment une discontinuité dans le paysage, propice à l'invasion de nouvelles espèces dites généralistes à large distribution (Alignier, 2010).
Les structures en « $\mathbf{J}$ inversé » des peuplements des différents groupes de relevés issus des habitats forestiers, suggère qu'il s'agit des formations stables (Ouédraogo, 2006). Cette stabilité traduit l'importance des individus de régénération. Les formes de la structure diamétrique en «J inversé » sont typiques pour la plupart à des forêts tropicales (Vroh et al., 2011). Au niveau des terres submergées, on a observé des relevés issus des espaces cultivés, les jachères et les forêts sur terre ferme. Ces espaces abondent dans les alentours de la Forêt des Marais Tanoé-Ehy et sont rarement observés à l'intérieur (Abrou et al., 2017). En effet, dans ces zones exondées l'activité anthropique est plus marquée du fait de la facilité d'accès. On retrouve des espèces héliophiles telles que Funtumia africana, Albizia ferruginea, Myrianthus arboreus, dans des jachères. Il s'agit d'espèces qui ont une forte capacité de dispersion et d'adaptation aux milieux perturbés (Vroh et al., 2011). Dans les plantations de cacaoyers et de palmiers, les espèces les plus importantes sont Cola nitida, Ricinodendron heudelotii, Milicia excelsa, Pycnanthus angolensis. Ces espèces sont généralement laissées délibérément par les paysans pour servir de produits de cueillette ou de bois d'œuvre. Cela est observé dans plusieurs zones de production de cacao en Côte d'Ivoire (Kpangui et al., 2015) en Afrique Centrale (Duguma et al., 2001). Dans ces plantations, la faible richesse spécifique s'expliquerait par les techniques culturales utilisées par les populations locales. En effet, au niveau de la cacaoculture, la plupart des paysans utilisent la variété «Ghana ». Cette variété ne nécessite pas d'ombrage (Kpangui et al., 2015) d'où la faible densité des espèces associées au cacaoyer. Ce constat a été aussi fait au niveau des plantations de palmiers. En général, dans la zone d'étude au cours de la première année, le palmier est planté avec des cultures telles que le manioc, la banane plantain, le cacao, le café ou le maïs. Lorsque les palmiers deviennent assez grands, leurs cimes deviennent jointives empêchant la lumière d'atteindre le sol pour favoriser la 
régénération de la plupart d'espèces arborescentes héliophiles.

La forme de l'histogramme des tiges dans les espaces dont l'activité anthropique est moindre montrent que ceux-ci sont faiblement perturbés (G4) comparativement aux espaces cultivés (G5) qui sont très pertubés. En effet, dans les jachères (G4) l'on note un faible taux d'espèces de grands diamètres. Cet état de fait s'expliquerait par l'abattage des individus de grands diamètre pour l'installation de la culture. La plupart de ces jachères sont des espaces anciennement cultivés de palmiers et de cocotiers. Les travaux de Marien et Mallet (2004), au Parc National d'Azagny avaient fait état de cette situation. En ce qui concerne les espaces cultivés (plantations de cacaoyer et palmiers), le nombre élevé d'individus de grands diamètres est très marqué. Cet état de fait s'expliquerait par la présence des espèces telles que Cola nitida, Ricinodendron heudelotii, Milicia excelsa, Pycnanthus angolensis, car arbitrairement préservés par les paysans pour leur importance socioéconomique.

\section{Conclusion}

La présente étude a permis de faire un bilan du niveau de diversité de la flore et de la végétation des communautés végétales de l'espace de la Forêt des Marais Tanoé-Ehy. Sur l'ensemble des cinq communautés végétales échantillonnées dans l'espace FMTE, 568 espèces végétales ont été recensées. Cette étude a permis de déceler cinq groupes de relevés permettant de donner des informations sur l'abondance des taxons dans ces relevés et sur la physionomie du biotope. Des espèces indicatrices des milieux inondés et exondés varient d'une communauté à une autre. Ainsi, elles peuvent constituer des indicateurs clés pour le suivi environnemental de la FMTE. Les forêts sur terre ferme situées généralement à la lisière de la FMTE sont les plus diversifiées et les plus riches en espèces. Les forêts marécageuses et celles situées sur terre périodiquement inondée, bien que stable, sont moins favorables à l'installation de la plupart des espèces rencontrées sur terre ferme. Les jachères bien qu'étant des habitats instables comme des plantations de cacaoyers et de palmiers, ont une richesse et une diversité assez élevées qui témoignent de la rapidité de la reconstitution de la végétation après des activités agricoles. Les plantations de palmiers et de cacaoyers sont relativement pauvres du fait du manque d'encadrement des paysans pour la pratique de systèmes agroforestiers.

Dans l'espace de la FMTE, des activités d'enrichissement des plantations ont été entreprises depuis 2014 par l'ONG Action pour la Conservation de la Biodiversité en Côte d'Ivoire (ACB-CI) et se poursuivent. Les principales espèces mise en culture par cet organisme sont Ricinodendron heudelotii, Garcinia cola, Irvingia gabonensis, Xylopia aethiopica et Cola nitida. Les individus plantés de ces espèces, étaient encore petits au moment de la réalisation de cette étude. Leur croissance et développement futurs pourront permettre d'enrichir les parcelles agricoles et donc de servir de modèle pour une agriculture durable dans l'espace de la FMTE. Ainsi, le FMTE sera préservée de l'isolement et des pressions exercées par les populations à sa périphérie.

\section{CONFLIT D'INTERETS}

Les auteurs de cet article déclarent qu'il n'y a aucun conflit d'intérêts.

\section{CONTRIBUTIONS DES AUTEURS}

JENA a contribué à la collecte des données, à la conception et à la structuration de l'étude, à la recherche documentaire et à la rédaction de l'article. DK a participé à la correction du manuscrit. CYAY a contribué à la conception, à la structuration de l'étude et a supervisé la rédaction du manuscrit.

\section{REMERCIEMENTS}

La présente étude a aussi bénéficié de l'appui technique d'un programme du Centre Suisse de Recherches Scientifiques (CSRS) intitulé Recherche et Actions pour la Sauvegarde des Primates en Côte d'Ivoire 
(RASAPCI). Le bon déroulement des travaux au sein de la Forêt des Marais Tanoé-Ehy a été aussi possible grâce à la collaboration des populations locales des Sous-préfectures de Nouamou et Noé.

\section{RÉFÉRENCES}

Abrou NEJ, Kpangui KB, Vroh BTA, Adou Yao CY. 2017. Déterminismes de la Dynamique de la Forêt des Marais Tanoé-Ehy (FMTE). European Scientific Journal, 273(13): 301-317. DOI : http://dx.doi.org/10.19044/esj.2017.v13n 27p301

Adou Yao CY, Béné J-C, Kouamé D, Kpangui KB. 2007. Inventaire préliminaire de la flore et description de la végétation de la forêt des marais Tanoé. Rapport pour RASAP-CI, Abidjan, Côte d'Ivoire, 29 p.

Aké-Assi L. 2001. Flore de la Côte d'Ivoire 1, catalogue, systématique, biogéographie et écologie. Conservatoire et Jardin Botanique de Genève: Genève, Suisse, Boisseria, 57, tome 1; $396 \mathrm{p}$.

Aké-Assi L. 2002. Flore de la Côte d'Ivoire 2, catalogue, systématique, biogéographie et écologie. Conservatoire et Jardin Botanique de Genève: Genève, Suisse, Boisseria, 58, tome 2; $441 \mathrm{p}$.

Alignier A. 2010. Distribution des communautés végétales sous l'influence des lisières forestières dans des bois fragmentés. Thèse: Institut National Polytechnique de Toulouse, $239 \mathrm{p}$.

APG IV. 2016.- An update of the Angiosperm Phylogeny Group classification for the orders and families of flowering plants. Botanical Journal of the Linnean Society, 181: 1-20.

Barnaud G, Fustec E. 2007. Conserver les zones humides: Pourquoi? Comment? ÉditionQuae, $230 \mathrm{p}$.

Baize D, Jabiol B. 2011. Guide pour la description des sols. Editions Quae, Collection Savoir-Faire, 978-2-75921034-3, 429 p. 〈hal-01195043〉.

Brou YT, Akindès F, Bigot S. 2005. La variabilité climatique en Côte d'Ivoire: entre perceptions sociales et réponses agricoles. Cahiers Agricultures, 14: 533540.

Burel F, Baudry J. 2001. Écologie du paysage. Concepts, méthodes et applications. Annales de Géographie, t110, 618, 201.

Dufrêne M. 2004. The IndVal program version 2.0. http://mrw.wallonie.be/dgrne/sibw/outils /home.html. Consulté en Décembre 2015.

Duguma B, Gockowski J, Bakala J. 2001. Smallholder cacao (Theobroma cacao Linn.) cultivation in agroforestry systems of West and Central Africa: challenges and opportunities.Agroforestry Systems, 51: 177-188.

Egnankou WM. 2015. Flore et végétation des zones humides du Sud-Est de la Côte d'Ivoire: problème de pollution et méthode de lutte contre les végétaux aquatiques envahissants (VAE). Thèse de Doctorat d'Etat ès Sciences Naturelles (Botanique), Université Félix Houphouët-Boigny, Côte d'Ivoire, 125 p.

Gnagbo A, Kpangui KB, Adou Yao CY. 2015. Distribution des épiphytes de Côte d'Ivoire: effets des zones phytogéographiques et des variations pluviométriques. Afrique SCIENCE, 11(1): $\quad$ 175-186. http://www.afriquescience.info

Guillaumet JL, Adjanohoun E. 1971. La végétation de la Cote d'Ivoire. In Le Milieu Naturel de Côte d'Ivoire. Mémoires ORSTOM : Paris (France), 50, 161-263.

Hussain F, Shah SM, Sher H. 2007. Traditional resource evaluation of some plants of Mastuj, District Chitral, Pakistan. Pakistan Journal of Botany, 66: 1011-1029. http://www.parc.gov.pk/NARC/narc.htm 1

Koné I, Béné J-CK, N'Guessan AK, Bitty AE, Koffi AD, Akpatou KB, Gonédélé BS. 2008. Plaidoyer pour la sauvegarde de la Forêt des Marais Tanoé (Sud-est de la Côte d'Ivoire), un site à valeur de conservation exceptionnelle en Afrique de l'Ouest et dans le monde. RASAPCI. Abidjan, Côte d'Ivoire, 25 p. 
Kpangui KB, Kouamé D, Goné BZB, Vroh BTA, Koffi BJ, Adou Yao CY. 2015. Typology of cocoa-based agroforestry systems in a forest-savannah transition zone: case study of Kokumbo (Centre, Côte d'Ivoire). International Journal of Agronomy and Agricultural Research, 3(6): 36-47. http://www.innspub.net/

Lebrun JP, Stork AL. 1997. Énumération des plantes à fleurs d'Afrique tropicale: 4. Gamopétales : Clethraceae à Lamiaceae. Conservatoire et Jardin Botaniques de Genève ; $712 \mathrm{p}$.

Levison J, Larocque M, Fournier V, Gagné S, Pellerin S, Ouellet MA. 2013. Dynamics of a headwater system and peatland under current conditions and with climate change Hydrological Processes, 27(19). DOI: 10.1002/hyp.9978

Marien JN, Mallet B. 2004. Nouvelles perspectives pour les plantations forestières en Afrique centrale. Bois et Forêts des Tropiques, (282): 67-79. DOI :

https://doi.org/10.19182/bft2004.282.a20 221

Masharabu T, 2011. Flore et végétation du Parc National de la Ruvubu au Burundi : diversité, structure et implications pour la conservation, Thèse de doctorat, Université Libre de Bruxelles, Belgique, $224 \mathrm{p}$.

Missa K, Ouattara D, Koné M, Bakayoko A. 2015. Etude floristique et diversité de la forêt des Marais Tanoé-Ehy (Sud-Est de la Côte d'Ivoire). Journal of Animal \& Plant Sciences, 25(3): 3917-3938. http://www.m.elewa.org/JAPS

Moore D, Coste D. 2006. Plurilinguismes et Ecole. Editions Didier, collection LAL : Paris ; $320 \mathrm{p}$.

Nshimba SM, Ndjele MB, Lejoly J. 2005. Etude de la répartition spatiale des espèces dominantes dans la forêt marécageuse de l'île Mbiyeà Kisangani, R.D.Congo. Résumé aetfat Yaoundé. $272 \mathrm{p}$.

Ouédraogo A. 2006. Diversité et dynamique de la végétation ligneuse de la partie orientale du Burkina Faso. Thèse de doctorat, Université de Ouagadougou, $196 \mathrm{p}$.

Sangne CY, Barima YSS, Bamba I, N'Doumé CTA. 2015. Dynamique forestière postconflits armés de la Forêt classée du Haut-Sassandra (Côte d'Ivoire). VertigO, 15(3) : 1-18. DOI : https://id.erudit.org/iderudit/1035879ar

Tiébré MS, Ouattara D, Kpangui KB, Kouassi DF, N'Guessan KE. 2016a. Diversité floristique de la région de Foungbesso en zone de transition forêt - savane à l'ouest de la Côte d'Ivoire. International Journal of Biological and Chemical Sciences, 10(3): 1007-1016. DOI: http://dx.doi.org/10.4314/ijbcs.v10i3.8

Tiébré MS, Ouattara D, Kpangui KB, Gnagbo A, N'Guessan KE. 2016b. Diversité floristique et disponibilité des plantes utilitaires en zone soudanienne de la Côte d'Ivoire. Journal of Applied Biosciences, 102: 9699 - 9707. DOI: http://dx.doi.org/10.4314/jab.v102i1.4

Vroh BTA, Kouame NF, Tondoh EJ. 2011. Etude du potentiel de restauration de la diversité floristique des agrosystèmes de bananiers dans la zone de Dabou (Sud Côte d'Ivoire). Sciences \& Nature, 1(8): $37-52$.

Zadou DA, Koné I, Mouroufié VKA, Adou Yao CY, Gleanou EK, Kablan YA, Coulibaly D, Ibo J. 2011. Valeur de la forêt des Marais Tanoé-Ehy (Sud Est de la Côte d'Ivoire) pour la conservation: dimension socio-anthropologique. Tropical Conservation Sciences, 4 (4) : 373-385. DOI : https://doi.org/10.1177\%2F19400829110 0400402

Zedler JB, Kercher S. 2005. Wetland resources: status, trends, ecosystem services, and restorability. Annual Review of Environment and Resources, (30): 39-74. DOI: 10.1146/annurev.energy.30.050504.1442 48. 